\title{
From diarrhoea to haemolytic uraemic syndrome - when to seek advice
}

It is difficult to distinguish between infectious and non-infectious bloody diarrhoea, and between infections with verocytotoxin-producing Escherichia coli (VTEC), such as E. coli O157, and other pathogens. Typically, $10-15 \%$ of VTEC infections quickly progress to haemolytic uraemic syndrome (HUS) (Tarr et al., 2005). To prevent this, cases must be identified more rapidly than possible by microbiological diagnosis. If achieved, this is advantageous for two reasons. Firstly, intravenous volume expansion, even before VTEC is microbiologically identified in such patients, might mitigate the risk of developing oligoanuric forms of HUS which are associated with poorer outcome (Ake et al., 2005). Secondly, secondary spread of VTEC organisms, which is common and occurs early in the course of disease, might be reduced by hospitalizing the paediatric patient or otherwise separating the patient from its sibling (Werber et al., 2008).

If a child presents to primary care with diarrhoea, a careful history can assist in differentiating an infectious from a noninfectious aetiology. Infant colitis is almost invariably restricted to children under 6 months of age (Xanthakos et al., 2005), necrotizing enterocolitis is largely limited to neonatal intensive care units (Ostlie et al., 2003), and inflammatory bowel disease usually presents with a history of symptoms over several months (Spray et al., 2001). In contrast, bloody diarrhoea caused by bacterial pathogens usually occurs fulminantly in older, previously healthy, children. Questions that might suggest infectious aetiology for the child's illness include:

- Has the child been in contact with another case of diarrhoea or bloody diarrhoea? An associated case would support an infectious aetiology but its absence does not exclude one.

- Has the child had any exposure that might plausibly suggest an infectious aetiology? The absence of such an exposure does not exclude infection but its presence might make one more likely. For instance, contact with farm animals increases the probability of zoonotic infection, especially with VTEC (Keen et al., 2006).

- Has the child been well in recent weeks? Infectious diarrhoea is usually acute, non-infectious diarrhoea less frequently so.

- How severe is the abdominal pain? Pain out of proportion to the severity of the diarrhoea is more typical of an infectious aetiology (Klein et al., 2002).

- Has the child had a fever? Children with VTEC infections are frequently afebrile at presentation but up to half give a history of fever prior to presentation (Wong et al., 2000).

- How much blood is there in the stool? If present, is blood intermixed with liquid stool, or streaked on a solid stool's surface? The latter suggests a benign rectal bleed.

Acute bloody diarrhoea is a medical emergency at all ages but especially in children under the age of 15 years. Recently, Murphy (2008) provided a detailed approach to the management of bloody diarrhoea in children in primary care. He addressed bloody diarrhoea from confirmed infectious and non-infectious causes. While noting that in the developed world bloody diarrhoea in children is 15-20 times more likely to be caused by intestinal infections than by inflammatory bowel disease, he did not address how to infer a probably infectious aetiology or how to manage one before microbiological confirmation.

It is important for anyone assessing cases of bloody diarrhoea to have a low threshold of suspicion for VTEC infection as it is the commonest cause of HUS, an important cause of childhood renal failure, worldwide. A clinician who suspects VTEC infection may wish to contact the local Health Protection team and enquire whether other cases of suspected or confirmed VTEC have been reported locally. He/she should send a stool sample for microbiological identification of pathogens, and culture-based diagnosis (not toxin-based screening) should be requested. If the clinician is in doubt about the child's condition, he/she should contact a consultant paediatrician or infectious disease physician for advice on possible admission to hospital, for intravenous volume expansion and infection control (Ake et al., 2005). An algorithm (Fig. 1) is intended to aid the primary care physician in such situations.

\section{Acknowledgements}

The Scottish VTEC forum is acknowledged for their valuable input into the manuscript.

Kevin G. J. Pollock, ${ }^{1}$

Alexander Stewart, ${ }^{2}$ T. James Beattie, ${ }^{3}$ W. T. Andrew Todd, ${ }^{4}$ Christina K. Ahn, ${ }^{5}$ Phillip I. Tarr ${ }^{5}$ and John M. Cowden ${ }^{1}$

\section{${ }^{1}$ Health Protection Scotland, Clifton House, Clifton Place, Glasgow, UK \\ ${ }^{2}$ Coatbridge Health Centre, Coatbridge, UK \\ ${ }^{3}$ Renal Unit, Royal Hospital for Sick Children, Glasgow, UK \\ ${ }^{4}$ Infectious Diseases Unit, Monklands Hospital, Airdrie, UK \\ ${ }^{5}$ Department of Pediatrics, Washington University of St Louis, Missouri, USA}

Correspondence: Kevin G. J. Pollock (Kevin.Pollock@hps.scot.nhs.uk)

Ake, J. A., Jelacic, S., Ciol, M. A., Watkins, S. L., Murray, K. F., Christie, D. L., Klein, E. J. \& Tarr, P. I. (2005). Relative nephroprotection during Escherichia coli O157:H7 infections: association with intravenous volume expansion. Pediatrics 115, e673-e680. 
Patient presents with acute diarrhoea

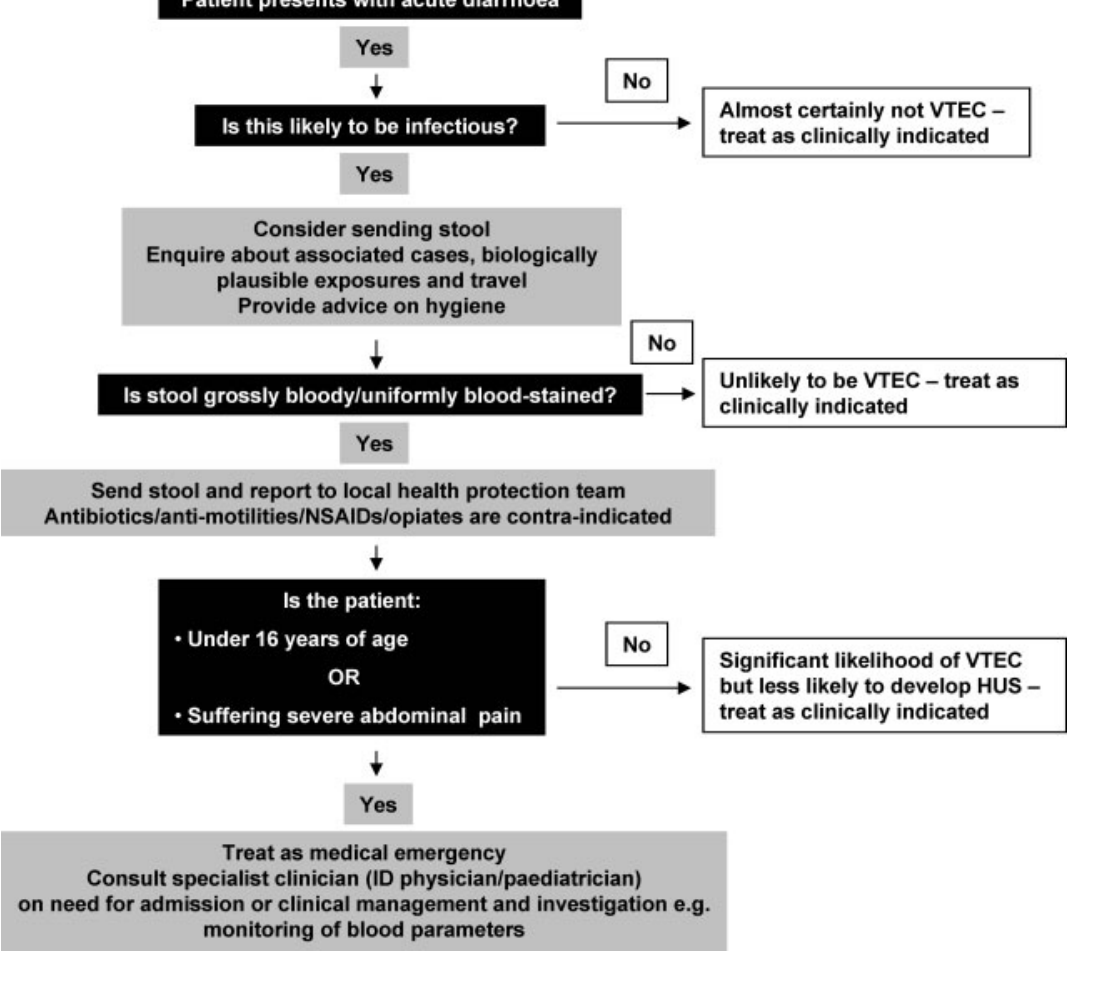

Fig. 1. Early clinical management of suspected VTEC infection.

Keen, J. E., Wittum, T. E., Dunn, J. R., Bono, J. L. \& Durso, L. M. (2006). Shiga-toxigenic Escherichia coli $\mathrm{O} 157$ in agricultural fair livestock, United States. Emerg Infect Dis 12, 780-786.
Klein, E. J., Stapp, J. R., Clausen, C. R., Boster, D. R., Wells, J. G., Qin, X., Swerdlow, D. L. \& Tarr, P. I. (2002). Shiga toxin-producing Escherichia coli in children with diarrhea: a prospective point-of-care study. J Pediatr 141, 172-177.

Murphy, M. S. (2008). Management of bloody diarrhoea in children in primary care. BMJ 336 , 1010-1015.

Ostlie, D. J., Spilde, T. L., St Peter, S. D., Sexton, N., Miller, K. A., Sharp, R. J., Gittes, G. K. \& Snyder, C. L. (2003). Necrotising enterocolitis in full-term infants. J Pediatr Surg 38, 1039-1042.

Spray, C., Debelle, G. D. \& Murphy, M. S. (2001). Current diagnosis, management and morbidity in paediatric inflammatory bowel disease. Acta Paediatr 90, 400-405.

Tarr, P. I., Gordon, C. A. \& Chandler, W. L. (2005). Shiga-toxin-producing Escherichia coli and haemolytic uraemic syndrome. Lancet 365 , 1073-1086.

Werber, D., Mason, B. W., Evans, M. R. \& Salmon, R. L. (2008). Preventing household transmission of Shiga toxin-producing Escherichia coli O157 infection: promptly separating siblings might be the key. Clin Infect Dis 46, 1189-1196.

Wong, C. S., Jelacic, S., Habeeb, R. L., Watkins, S. L. \& Tarr, P. I. (2000). The risk of the hemolytic-uremic syndrome after antibiotic treatment of Escherichia coli O157:H7 infections. N Engl J Med 342, 1930-1936.

Xanthakos, S. A., Schwimmer, J. B., MelinAldana, H., Rothenberg, M. E., Witte, D. P. \& Cohen, M. B. (2005). Prevalence and outcome of allergic colitis in healthy infants with rectal bleeding: a prospective cohort study. J Pediatr Gastroenterol Nutr 41, 16-22. 